\title{
OBTENCIÓN DEL MODELO CINEMÁTICO INVERSO DE SISTEMAS ROBOTIZADOS DE CADENA CINEMÁTICA ABIERTA EMPLEANDO BASES DE GROEBNER. APLICACIÓN A UN ROBOT HEXÁPODO
}

\author{
José Guzmán-Giménez, Ángel Valera Fernández \\ Instituto de Automática e Informática Industrial (ai2), Universitat Politècnica de València, \\ Camí de Vera s/n, 46022 Valencia, España. joguz@upvnet.upv.es, giuprog@isa.upv.es \\ Vicente Mata Amela \\ Centro de Investigación de Ingeniería Mecánica, Universitat Politècnica de València, \\ Camí de Vera s/n, 46022 Valencia, España. vmata@mcm.upv.es \\ Miguel Ángel Díaz-Rodríguez \\ Escuela de Ingeniería Mecánica, Universidad de Los Andes, \\ Av. Alberto Carnevalli, Mérida 5101, Venezuela. dmiguel@ula.ve
}

\section{Resumen}

Uno de los elementos más importantes del control de un sistema robotizado es su Modelo Cinemático Inverso (IKM), el cual calcula las referencias de posición y velocidad requeridas para que el robot siga una trayectoria predeterminada. Los métodos comúnmente empleados para obtener el IKM de sistemas robotizados de cadena cinemática abierta dependen fuertemente de la geometría del robot estudiado y no son procedimientos sistemáticos. Este trabajo presenta el desarrollo de un procedimiento de cálculo del IKM de robots de cadena cinemática abierta utilizando Bases de Groebner. El procedimiento propuesto no depende de la geometría del robot, ofreciendo una metodología sistemática para el cálculo del IKM. Las entradas del procedimiento desarrollado son los parámetros Denavit-Hartenberg del robot, y ofrece como salidas los códigos que constituyen el IKM calculado. Estos códigos pueden emplearse directamente en el sistema de control del robot o para simular su funcionamiento. El desempeño del procedimiento desarrollado se comprobó calculando el IKM de un robot hexápodo. El tiempo de cómputo del IKM calculado es comparable con el requerido por el modelo obtenido por métodos tradicionales, y el error de las referencias que ofrece como salida es absolutamente despreciable en todo el espacio de trabajo del hexápodo analizado.

Palabras clave: Problema cinemático, Modelo Cinemático Inverso, Bases de Groebner, robot caminante hexápodo.

\section{INTRODUCCIÓN}

El diseño y modelado del sistema de control de un sistema robotizado comienza con la resolución de su problema cinemático, el cual se divide en dos partes: el cálculo de la cinemática directa del robot y la obtención de su Modelo Cinemático Inverso (también conocido como IKM por sus siglas en inglés).

La cinemática directa de un sistema robotizado es el sistema de ecuaciones con el cual se calcula la posición y orientación de un punto específico de la estructura del robot en función del estado actual de sus actuadores. Dicho punto normalmente es un punto importante de la estructura del robot, como el efector final en el caso de brazos manipuladores, o el centro de masa de un robot móvil. La cinemática directa es una pieza fundamental en el modelado de los movimientos del robot y también es importante para la obtención de su Modelo Cinemático Inverso (IKM).

Por otro lado, el IKM se encarga de determinar las referencias de control requeridas por los actuadores del robot, para que éste pueda llegar a una posición deseada o siga una trayectoria predeterminada, por lo que el IKM es una parte fundamental en el sistema de control del robot, como se presenta en la figura 1.

Existen diferentes procedimientos que permiten calcular la cinemática directa de un sistema robotizado de cadena cinemática abierta, entre los que encontramos el algoritmo de Denavit-Hartenberg [8][2], los cuaterniones duales [18][15] y el modelado del robot por medio de Matrices de Desplazamiento [3]. Todos estos procedimientos determinan la cinemática 


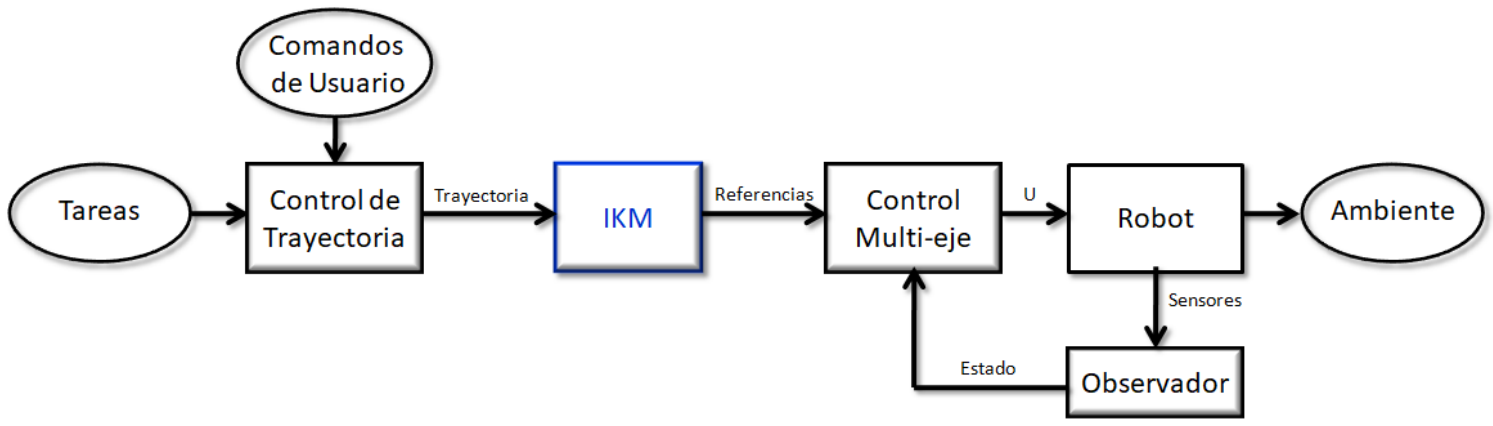

Figura 1: Modelo Cinemático Inverso (IKM) dentro del sistema de control del robot

directa del robot a través de la ejecución de una metodología sistemática, la cual es independiente de la complejidad de la estructura mecánica del robot o de su geometría.

Por otro lado, el problema cinemático inverso de los sistemas robotizados de cadena cinemática abierta presenta un mayor desafío, porque los métodos más comúnmente utilizados para calcular el IKM, el método geométrico y el analítico, dependen fuertemente de la geometría del robot analizado y no ofrecen un procedimiento sistemático para la obtención del resultado. El método geométrico consiste en descomponer el problema cinemático inverso del robot estudiado en varios problemas de geometría plana, y resolver dichos problemas empleando relaciones trigonométricas [2][4]. Por su propia definición, es obvio que este método depende muy fuertemente de la geometría de la estructura del robot, y cualquier secuencia de pasos empleada para obtener el IKM de un sistema robotizado no será válida para otra estructura diferente.

El método analítico para el cálculo del IKM consiste en despejar el vector de estado de los actuadores del robot del sistema de ecuaciones que define su cinemática directa $[18][16][5]$. El inconveniente de esta metodología radica en que el mencionado sistema de ecuaciones normalmente está compuesto por ecuaciones no lineales, por lo que dicho despeje no se puede efectuar empleando procedimientos tradicionales de álgebra matricial, sino que se deben buscar, dentro del sistema de ecuaciones estudiado, relaciones específicas para cada caso. Esto significa que el método analítico tampoco es un procedimiento sistemático, porque los pasos seguidos para calcular el IKM de un robot con una determinada estructura, no necesariamente sean válidos o aplicables para otro robot diferente.

Los inconvenientes anteriormente señalados han impulsado el desarrollo de diversos proyectos que emplean diferentes técnicas de inteligencia artificial para la resolución del problema cinemático inverso, como redes neuronales [14][11][7][20], algoritmos genéticos [13] y técnicas de enjambre de partículas [19]. Con estas técnicas se obtienen soluciones normalmente satisfactorias para el problema cinemático inverso de posición y orientación de sistemas robotizados, sin que la estructura geométrica del robot presente un inconveniente. Pero es importante destacar que estos métodos no determinan el Modelo Cinemático Inverso (IKM) del sistema robotizado, por lo que las referencias de posición que ofrecen como salida no provienen de una función diferenciable, y por consiguiente no son capaces de calcular las referencias de velocidades ni las de las aceleraciones requeridas por los diferentes grados de movilidad del robot. Además dichos métodos pueden incurrir en los conocidos problemas de entrenamiento de las técnicas de inteligencia artificial, como son el sobreentrenamiento de las redes neuronales o el "overfitting" de los algoritmos genéticos.

En la actualidad se están desarrollando proyectos en el campo de la robótica que aplican la teoría de Bases de Groebner para implementar métodos sistemáticos de resolución del problema cinemático que sean independientes de la geometría del robot. Entre estos proyectos destacan los trabajos de Kendricks [12], en los cuales determina el IKM de brazos manipuladores utilizando Bases de Groebner, así como los proyectos de Abbasnejad et al. [1], que usan esta teoría para el modelado y control de robots manipulados por cables. Rameau et al. [17] emplea las Bases de Groebner para calcular las condiciones de movilidad de diversos mecanismos que pueden emplearse en brazos manipuladores o robots paralelos. Los trabajos de Gan et al. [9] y de Huang et al. [10] utilizan la teoría de Bases de Groebner para la resolución del problema cinemático de robots paralelos, mientras que Uchida et al. [21] emplea esta teoría para la simulación dinámica de robots paralelos.

El objetivo de este trabajo consistió en emplear 
la teoría de Bases de Groebner para desarrollar un procedimiento sistemático de cálculo del IKM de sistemas robotizados de cadena cinemática abierta. Este procedimiento se explica en detalle en la sección 3, y fue utilizado para calcular el IKM de un robot caminante hexápodo. La sección 2 presenta el modelo cinemático del hexápodo, calculado por métodos clásicos, el cual fue empleado como modelo de referencia para analizar el desempeño del procedimiento desarrollado en este trabajo. La sección 4 presenta el análisis de este desempeño, comparando las salidas del IKM obtenido en este proyecto con las del modelo de referencia. Finalmente en la sección 5 se presentan las conclusiones de los resultados obtenidos.

\section{MODELO CINEMÁTICO CALCULADO POR EL MÉTODO GEOMÉTRICO}

El sistema robotizado con el que se trabajó en este proyecto, empleándolo como banco de pruebas para el procedimiento de cálculo del IKM desarrollado, es el robot caminante hexápodo BH3-R de la compañía Lynxmotion Inc., el cual se presenta en la figura 2.

Como el hexápodo presentado en la figura 2 tiene geometría circular, su problema cinemático inverso se puede resumir a hallar el IKM de una de las extremidades del robot, para posteriormente aplicar las transformaciones correspondientes entre el centro del robot y el origen de cada una de sus extremidades. Se seleccionó este robot porque tiene 3 grados de movilidad rotacionales para el posicionamiento, como la mayoría de los brazos manipuladores industriales y una gran cantidad de robots caminantes, y además es un sistema que sólo requiere de la resolución del problema cinemático de posicionamiento.

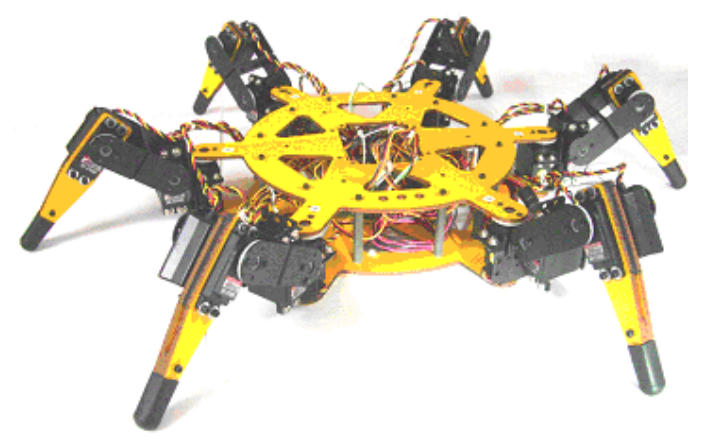

Figura 2: Robot hexápodo BH3-R de Lynxmotion Inc.

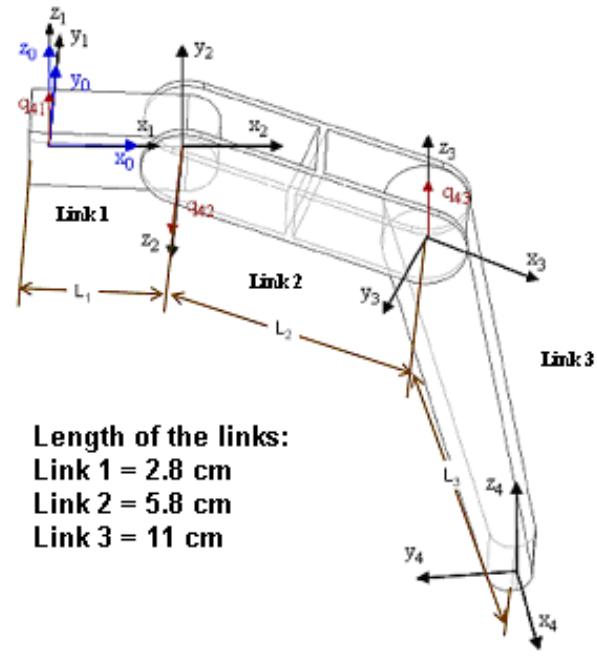

Figura 3: Diagrama de una de las extremidades del robot hexápodo

El primer paso para obtener el modelo cinemático de un robot por los métodos tradicionales consiste en analizar su cinemática directa.

\subsection{CINEMÁTICA DIRECTA DEL ROBOT HEXÁPODO}

Para la resolución del problema cinemático directo de una de las extremidades del hexápodo, presentado en la figura 2, se utilizó el método de Denavit-Hartenberg [2]. En la figura 3 se pueden observar los sistemas de coordenadas asignados durante la aplicación de este método, evidenciándose además los tres eslabones (links) que componen cada una de las extremidades del robot caminante.

A partir de los sistemas de coordenadas presentados en la figura 3, se pueden calcular los parámetros de Denavit-Hartenberg de la transformación entre el sistema de coordenadas del origen y el del final de la extremidad, los cuales se presentan en la tabla 1.

Tabla 1: Parámtetros de Denavit-Hatenberg de una extremidad del robot hexápodo

\begin{tabular}{|c|cccc|}
\hline Link & $\boldsymbol{\theta}$ & $\boldsymbol{d}$ & $\boldsymbol{a}$ & $\boldsymbol{\alpha}$ \\
\hline 1 & $q_{1}$ & 0 & $L_{1}$ & $\pi / 2$ \\
2 & $q_{2}$ & 0 & $L_{2}$ & $\pi$ \\
3 & $q_{3}+(\pi / 2)$ & 0 & $L_{3}$ & 0 \\
\hline
\end{tabular}

La ecuación 1 presenta la matriz de transformación entre el sistema de coordenadas del origen de la extremidad y el que se encuentra en su punto final, la cual se calcula siguiendo los pasos del método de Denavit-Hatenberg [2]. 


$$
A=\left[\begin{array}{cccc}
\cos \left(q_{1}\right) \sin \left(q_{2}-q_{3}\right) & -\cos \left(q_{1}\right) \cos \left(q_{2}-q_{3}\right) & -\sin \left(q_{1}\right) & \cos \left(q_{1}\right)\left[L_{1}+L_{2} \cos \left(q_{2}\right)+L_{3} \sin \left(q_{2}-q_{3}\right)\right] \\
\sin \left(q_{1}\right) \sin \left(q_{2}-q_{3}\right) & -\sin \left(q_{1}\right) \cos \left(q_{2}-q_{3}\right) & \cos \left(q_{1}\right) & \sin \left(q_{1}\right)\left[L_{1}+L_{2} \cos \left(q_{2}\right)+L_{3} \sin \left(q_{2}-q_{3}\right)\right] \\
-\cos \left(q_{2}-q_{3}\right) & -\sin \left(q_{2}-q_{3}\right) & 0 & L_{2} \sin \left(q_{2}\right)-L_{3} \cos \left(q_{2}-q_{3}\right) \\
0 & 0 & 0 & 1
\end{array}\right]
$$

\subsection{MODELO CINEMÁTICO INVERSO DEL ROBOT HEXÁPODO OBTENIDO A PARTIR DEL MÉTODO GEOMÉTRICO}

Antes de comenzar con la presentación del modelo cinemático inverso (IKM), calculado por el método geométrico, de la extremidad del robot hexápodo, es importante destacar que el sistema estudiado, al igual que ocurre con la mayoría de los brazos manipuladores, presenta hasta 4 soluciones posibles. Por consiguiente pueden existir hasta 4 configuraciones válidas para cada punto de la trayectoria deseada para dicha extremidad.

El primer paso del método geométrico para determinar el IKM de la extremidad del robot hexápodo (ver figura 3) consiste en expresar la posición angular del primer actuador de la extremidad, $q_{1}$, en función de la posición del extremo en el plano "XY", siguiendo las expresiones presentadas en 2. En esas expresiones se puede observar que hay 2 valores para $q_{1}$, $q_{1 f}$ y $q_{1 b}$, lo cual es debido a que 2 de las 4 posibles configuraciones de la extremidad se alcanzan cuando $q_{1}=q_{1 f}$, y las otras 2 con $q_{1}=$ $q_{1 b}$. Al final de esta sección se presentará la forma en la que se organizan estas 4 configuraciones.

$$
\begin{gathered}
q_{1 f}=\operatorname{atan} 2(y, x) \\
q_{1 b}=\operatorname{atan} 2(-y,-x)=q_{1 f}+\pi
\end{gathered}
$$

El segundo paso del método geométrico expresa las posibles soluciones de $q_{3}$ en función de los 4 posibles valores del ángulo $\beta$, como se presenta en 3 y 4.

$$
\begin{gathered}
q_{3 f u}=\beta_{f u}+\pi / 2 \\
q_{3 f d}=\beta_{f d}+\pi / 2 \\
q_{3 b u}=\beta_{b u}+\pi / 2 \\
q_{3 b d}=\beta_{b d}+\pi / 2 \\
\beta_{f u}=\operatorname{atan} 2\left(\sin \left(\beta_{f u}\right), \cos \left(\beta_{f u}\right)\right) \\
\beta_{f d}=-\beta_{f u} \\
\beta_{b d}=\operatorname{atan} 2\left(\sin \left(\beta_{b d}\right), \cos \left(\beta_{b d}\right)\right) \\
\beta_{b u}=-\beta_{b d}
\end{gathered}
$$

Las 4 posibles configuraciones del ángulo $\beta$, mostradas en 4 , se calculan a partir de las relaciones trigonométricas presentadas en 5 .

$$
\begin{gathered}
\cos \left(\beta_{f u}\right)=\frac{L_{2}^{2}+L_{3}^{2}-d_{f}^{2}}{2 \cdot L_{2} \cdot L_{3}} \\
\cos \left(\beta_{b d}\right)=\frac{L_{2}^{2}+L_{3}^{2}-d_{b}^{2}}{2 \cdot L_{2} \cdot L_{3}} \\
\sin \left(\beta_{f u}\right)=\sqrt{1-\cos \left(\beta_{f u}\right)^{2}} \\
\sin \left(\beta_{b d}\right)=\sqrt{1-\cos \left(\beta_{b d}\right)^{2}} \\
d_{f}=\sqrt{z^{2}+r_{f}^{2}} \\
d_{b}=\sqrt{z^{2}+r_{b}^{2}} \\
r_{f}=x \cdot \cos \left(q_{1 f}\right)+y \cdot \sin \left(q_{1 f}\right)-L_{1} \\
r_{b}=x \cdot \cos \left(q_{1 f}\right)+y \cdot \sin \left(q_{1 f}\right)+L_{1}
\end{gathered}
$$

Por último, las 4 configuraciones posibles para el último actuador que falta por resolver, $q_{2}$, son las presentadas en 6 .

$$
\begin{aligned}
q_{2 f u} & =\phi_{f}-\gamma_{f u} \\
q_{2 f d} & =\phi_{f}-\gamma_{f d} \\
q_{2 b u} & =\phi_{b}-\gamma_{b u} \\
q_{2 b d} & =\phi_{b}-\gamma_{b d}
\end{aligned}
$$

Las expresiones de las diferentes configuraciones de los ángulos $\phi$ y $\gamma$ se encuentran en 7 y 8 , respectivamente.

$$
\begin{gathered}
\phi_{f}=\operatorname{atan} 2\left(z, r_{f}\right) \\
\phi_{b}=\operatorname{atan} 2\left(z, r_{b}\right)
\end{gathered}
$$

$$
\begin{gathered}
\gamma_{f u}=\operatorname{atan} 2\left(\sin \left(\gamma_{f u}\right), \cos \left(\gamma_{f u}\right)\right) \\
\gamma_{f d}=-\gamma_{f u} \\
\gamma_{b d}=\operatorname{atan} 2\left(\sin \left(\gamma_{b d}\right), \cos \left(\gamma_{b d}\right)\right) \\
\gamma_{b u}=-\gamma_{b d} \\
\cos \left(\gamma_{f u}\right)=\frac{L_{2}^{2}+d_{f}^{2}-L_{3}^{2}}{2 \cdot L_{2} \cdot d_{f}} \\
\cos \left(\gamma_{b d}\right)=\frac{L_{2}^{2}+d_{b}^{2}-L_{3}^{2}}{2 \cdot L_{2} \cdot d_{b}} \\
\sin \left(\gamma_{f u}\right)=\sqrt{1-\cos \left(\gamma_{f u}\right)^{2}} \\
\sin \left(\gamma_{b d}\right)=\sqrt{1-\cos \left(\gamma_{b d}\right)^{2}}
\end{gathered}
$$

Finalmente, en 9 se presentan las estructuras de las 4 configuraciones que conforman el resultado final del modelo cinemático inverso del hexápodo estudiado. 


$$
\begin{array}{rlrl}
q_{f u} & =\left[\begin{array}{c}
q_{1 f} \\
q_{2 f u} \\
q_{3 f u}
\end{array}\right] & q_{f d}=\left[\begin{array}{c}
q_{1 f} \\
q_{2 f d} \\
q_{3 f d}
\end{array}\right] \\
q_{b u}=\left[\begin{array}{c}
q_{1 b} \\
q_{2 b u} \\
q_{3 b u}
\end{array}\right] & q_{b d}=\left[\begin{array}{c}
q_{1 b} \\
q_{2 b d} \\
q_{3 b d}
\end{array}\right]
\end{array}
$$

\section{PROCEDIMIENTO DE CÁLCULO DEL IKM EMPLEANDO BASES DE GROEBNER}

El procedimiento de cálculo del IKM desarrollado en este proyecto se divide en 5 pasos:

1. Solicitud de información

2. Cálculo de la cinemática directa del robot

3. Obtención del sistema de ecuaciones polinómicas

4. Selección del orden lexicográfico de las variables

5. Cálculo de las Bases de Groebner y obtención del IKM

Es importante destacar que estos pasos se ejecutan una única vez, fuera de línea, para calcular el IKM antes de poner el sistema robotizado en marcha, por lo que el tiempo de ejecución de estos pasos no influirá de ninguna forma en el tiempo de cómputo del IKM.

A continuación se detallan cada uno de los 5 pasos en los que está dividida la ejecución del procedimiento de cálculo del IKM de sistemas robotizados de cadena cinemática abierta.

\subsection{PRIMER PASO: SOLICITUD DE INFORMACIÓN}

El procedimiento comienza solicitando toda la información relevante sobre el robot analizado, para lo cual requiere que el usuario suministre únicamente dos datos: los parámetros de Denavit-Hartenberg del robot estudiado y los límites de sus grados de movilidad.

El primer dato, los parámetros de Denavit-Hartenberg del robot, es necesario para resolver el problema cinemático directo, el cual es un paso requerido para poder calcular las Bases de Groebner que constituirán el IKM. Los parámetros de Denavit-Hartenberg representan la información fundamental de cualquier sistema robotizado que se desee analizar, por lo que este dato es también indispensable para todos los métodos clásicos de resolución del problema cinemático.

Las limitaciones de los grados de libertad son empleadas para destacar las soluciones que realmente son alcanzables por el sistema analizado. De esta forma el IKM obtenido es capaz de desechar aquellas soluciones que estén fuera del espacio de trabajo del robot.

\subsection{SEGUNDO PASO: CÁlCULO DE LA CINEMÁTICA DIRECTA DEL ROBOT}

El segundo paso consiste en calcular la cinemática directa del robot a partir de los parámetros de Denavit-Hartenberg suministrados anteriormente. Dicho cálculo se realiza a través de la aplicación del método de Denavit-Hartenberg [2], por lo que esta parte del procedimiento es similar a la presentada en la sección 2.1 para el caso del robot hexápodo.

El resultado de este segundo paso es la matriz de transformación entre el sistema de coordenadas del origen del robot estudiado y el que se encuentra en su punto o efector final. De esa forma, al aplicar el procedimiento desarrollado sobre el sistema presentado en la figura 3 , el resultado de este paso será la misma matriz de transformación presentada en la ecuación 1 .

\subsection{TERCER PASO: OBTENCIÓN DEL SISTEMA DE ECUACIONES POLINÓMICAS}

Una vez calculada la cinemática directa del sistema estudiado, de la matriz de transformación obtenida se pueden extraer las ecuaciones que determinan la posición del extremo final del robot. Estas ecuaciones corresponden a los tres primeros elementos de la cuarta columna de la matriz de transformación, por lo que siguiendo con el ejemplo del robot hexápodo, se obtendrían las ecuaciones presentadas en 10 .

$$
\begin{gathered}
x=\cos \left(q_{1}\right)\left[L_{1}+L_{2} \cos \left(q_{2}\right)+L_{3} \sin \left(q_{2}-q_{3}\right)\right] \\
y=\sin \left(q_{1}\right)\left[L_{1}+L_{2} \cos \left(q_{2}\right)+L_{3} \sin \left(q_{2}-q_{3}\right)\right] \\
z=L_{2} \sin \left(q_{2}\right)-L_{3} \cos \left(q_{2}-q_{3}\right)
\end{gathered}
$$

Como se puede observar en 10, el resultado final de la cinemática directa son tres ecuaciones trigonométricas que determinan la posición del efector final del robot con respecto al sistema de coordenadas ubicado en su base, y es a partir de estas ecuaciones que comienza el cálculo del IKM empleando Bases de Groebner. 
Esas 3 ecuaciones se unen con las 3 respectivas identidades trigonométricas de la forma $\sin \left(q_{i}\right)^{2}+$ $\cos \left(q_{i}\right)^{2}=1$, para conformar un sistema de seis ecuaciones, en el cual las incógnitas son los senos $\mathrm{y}$ cosenos de los tres grados de movilidad de posicionamiento del robot, $q_{1}, q_{2}$ y $q_{3}$. Siguiendo con el ejemplo del robot hexápodo, el resultado de aplicar este paso del procedimiento sería el sistema de ecuaciones presentado en 11.

$$
\begin{gathered}
c_{1}\left[L_{1}+L_{2} c_{2}+L_{3} s_{2} c_{3}-L_{3} c_{2} s_{3}\right]-x=0 \\
s_{1}\left[L_{1}+L_{2} c_{2}+L_{3} s_{2} c_{3}-L_{3} c_{2} s_{3}\right]-y=0 \\
L_{2} s_{2}-L_{3} c_{2} c_{3}-L_{3} s_{2} s_{3}-z=0 \\
s_{1}^{2}+c_{1}^{2}-1=0 \\
s_{2}^{2}+c_{2}^{2}-1=0 \\
s_{3}^{2}+c_{3}^{2}-1=0
\end{gathered}
$$

\subsection{CUARTO PASO: SELECCIÓN DEL ORDEN LEXICOGRÁFICO DE LAS VARIABLES}

$\mathrm{Al}$ sistema de ecuaciones polinómicas multivariable resultante de la sección 3.3 se le puede calcular sus Bases de Groebner para obtener un sistema de ecuaciones triangular equivalente, cuya resolución es más simple. La solución de las Bases de Groebner calculadas es la misma que la del sistema de ecuaciones del que se parte [6], por lo que la obtención de estas bases permite simplificar el cálculo del IKM.

Es importante asignar un orden lexicográfico al momento de calcular las Bases de Groebner de un sistema de ecuaciones, ya que este orden determinará la forma en que se ordenan las ecuaciones en el sistema triangular final. En este proyecto se decidió utilizar el orden lexicográfico mostrado en 12, el cual representa el mismo orden en el que se obtienen los resultados en el método geométrico presentado en la sección 2.2.

$$
s_{2}>c_{2}>s_{3}>c_{3}>s_{1}>c_{1}
$$

\subsection{QUINTO PASO: CÁLCULO DE LAS BASES DE GROEBNER Y OBTENCIÓN DEL IKM}

Una vez seleccionado el órden lexicográfico de las variables, se procede al cálculo de las Bases de Groebner del sistema de ecuaciones polinómicas obtenido en la sección 3.3. Para el cálculo de las Bases de Groebner se emplea el algoritmo Buchberger, cuya explicación completa, al igual que la forma de emplear este algoritmo para calcular las Bases de Groebner de un sistema de ecuaciones polinómicas, se encuentra en [6]. El cálculo de las Bases de Groebner en este proyecto se realizó con la ayuda de Maple ${ }^{\mathrm{TM}} 16$.

Las Bases de Groebner que se obtienen como resultado constituyen un sistema de ecuaciones triangular, que se puede resolver de forma escalonada. Al resolver dicho sistema de ecuaciones triangular se obtiene el resultado para las 6 incógnitas del sistema de ecuaciones polinómicas original, las cuales corresponden a los valores de los senos y cosenos de los grados de movilidad del robot estudiado. Por consiguiente, para culminar el cálculo de la cinemática inversa del robot, sólo es necesario determinar el valor final de cada uno de estos grados de movilidad, empleando la expresión correspondiente de la forma presentada en 13 .

$$
q_{i}=\operatorname{atan} 2\left(s_{i}, c_{i}\right)
$$

El último paso del procedimiento desarrollado consiste en convertir el sistema de ecuaciones triangular obtenido, así como las correspondientes ecuaciones de resolución mostradas en 13, en los códigos que implementen el IKM final. El presente trabajo permite obtener estos códigos en 2 lenguajes diferentes: $\mathrm{C}++\mathrm{y}$ script ejecutable MATLABR. Los códigos del IKM se generan de forma automática gracias a los comandos de optimización y generación de código de Maple ${ }^{\mathrm{TM}}$ 16, y pueden ser empleados directamente en el sistema de control del robot o para simular su comportamiento.

\section{ANÁLISIS DEL DESEMPEÑO}

El procedimiento presentado en la sección 3 fue empleado para calcular el IKM de la extremidad del hexápodo presentada en la figura 3. Como se explicó en la sección anterior, la salida de este procedimiento son los códigos que definen este IKM, tanto en lenguaje $\mathrm{C}++$ como en script ejecutable de MATLABR.

Utilizando el código del script de MATLABß, se simuló el desempeño del IKM obtenido, probando su capacidad para resolver el problema cinemático inverso en todo el espacio de trabajo de la extremidad del robot hexápodo.

Las soluciones obtenidas de la simulación de este IKM fueron comparadas con las del modelo obtenido por el método geométrico, considerado el modelo de referencia, cuyas ecuaciones se presentaron en la sección 2.2 .

La figura 4 presenta los resultados de la comparación, en todo el espacio de trabajo, entre 

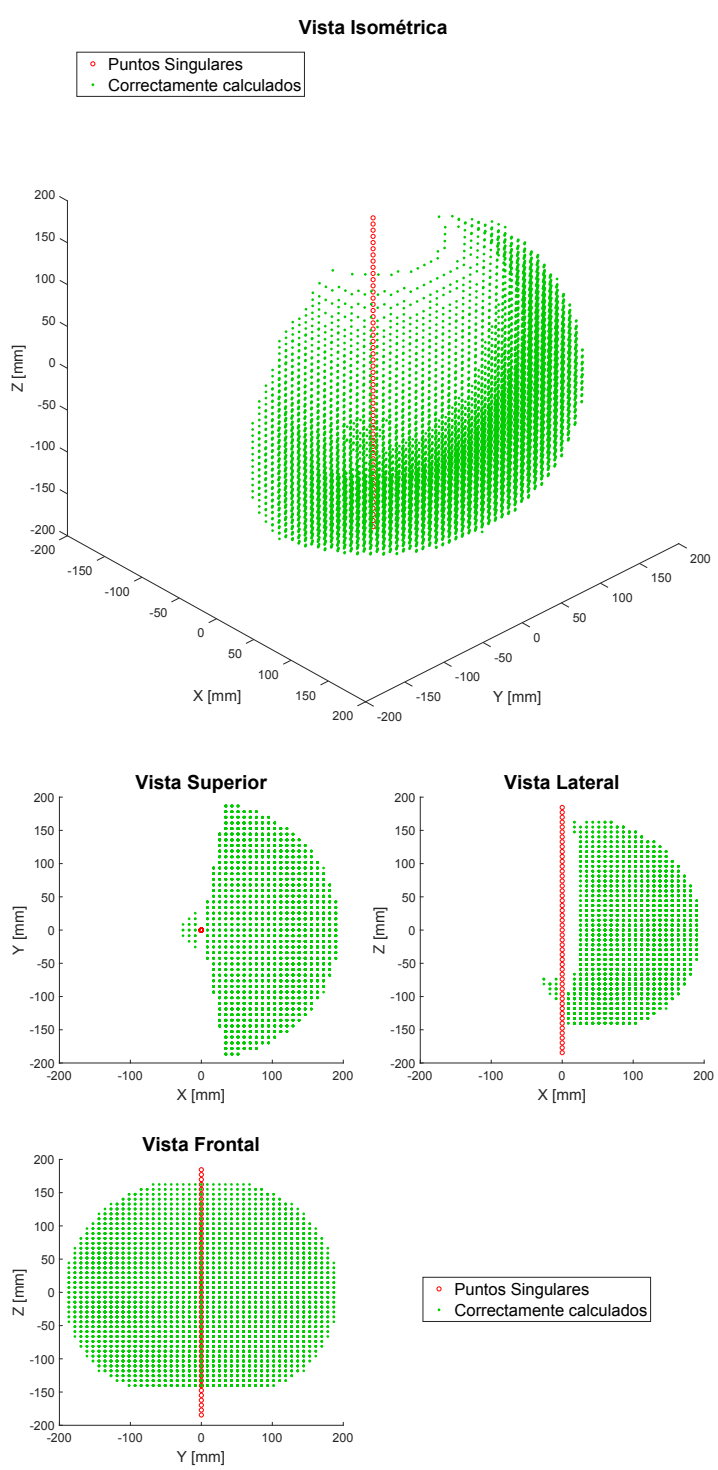

Figura 4: Análisis del desempeño del IKM calculado en todo el espacio de trabajo de la extremidad del robot hexápodo

el IKM calculado en este proyecto y el modelo de referencia. En dicha figura se marcan en verde (correctamente calculados) todas aquellas posiciones en las que el IKM analizado obtenga la misma cantidad de soluciones que el modelo de referencia y que, para todas esas soluciones, el error cuadrático medio (RMS) en el espacio de configuración sea menor a $1 \cdot 10^{-8}$.

En la figura 4 se observa como el IKM obtenido con nuestro procedimiento calcula satisfactoriamente todas las posibles soluciones dentro del espacio de trabajo de la extremidad del hexápodo, y además también determina correctamente todos los puntos singulares que el modelo de referencia identifica en el eje definido por la intersección de planos $X=0 \cap Y=0$.

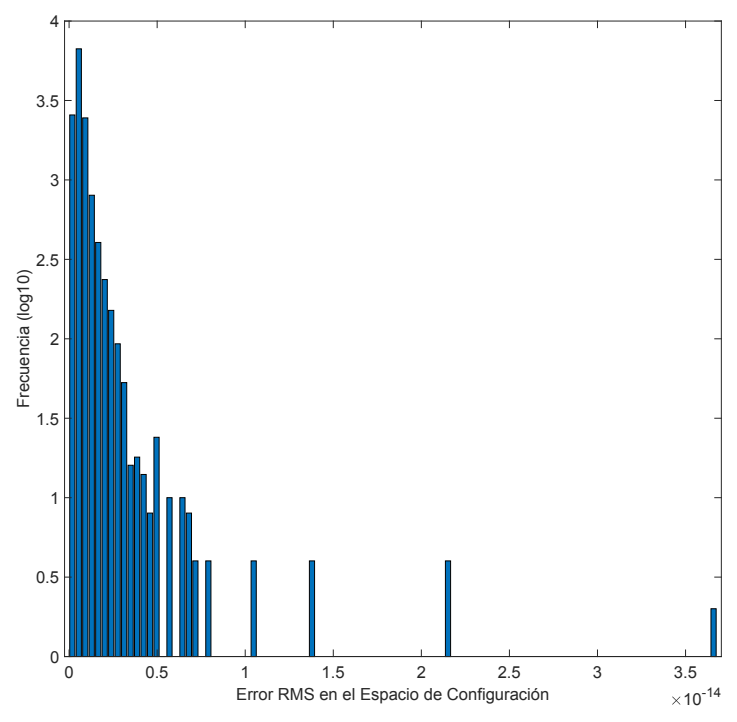

Figura 5: Histograma del error RMS para todos los puntos dentro del espacio de trabajo de la extremidad del hexápodo

La figura 5 presenta el histograma del error cuadrático medio (RMS) de todos los puntos analizados en el espacio de trabajo de la extremidad del hexápodo. En dicho histograma se puede observar que los errores de todas las soluciones calculadas por nuestro IKM son totalmente despreciables.

Finalmente la tabla 2 presenta la comparación de los tiempos de cómputo requeridos por el IKM calculado en este proyecto ("IKM") y por el modelo de referencia ("Ref"), observándose que estos tiempos son bastante similares, por lo que es totalmente equivalente utilizar nuestro IKM en lugar del modelo de referencia.

Tabla 2: Tiempos de cómputo requeridos por el IKM y por el modelo de referencia

\begin{tabular}{|c|ccc|}
\hline Mod & Min [s] & Prom [s] & Max [s] \\
\hline Ref. & $6.1 \mathrm{e}-6$ & $3.1 \mathrm{e}-5$ & $2.8 \mathrm{e}-3$ \\
IKM & $8.9 \mathrm{e}-6$ & $5.4 \mathrm{e}-5$ & $4.9 \mathrm{e}-3$ \\
\hline
\end{tabular}

\section{CONCLUSIONES}

En el presente proyecto se desarrolló un procedimiento que permite calcular, de forma sistemática, el IKM para el posicionamiento de sistemas robotizados de cadena cinemática abierta, sin que se requieran conocimientos especiales de la estructura geométrica del robot, más allá de sus parámetros de Denavit-Hartenberg. Este procedimiento, presentado en la sección 3, calcula el IKM 
mediante la aplicación de la teoría de Bases de Groebner, ofreciendo como resultado los códigos que definen dicho IKM, tanto en lenguaje $\mathrm{C}++$ como en script ejecutable para MATLABß. Los códigos obtenidos como salida pueden emplearse directamente en el sistema de control del robot, para determinar las referencias requeridas por los actuadores, o para la simulación de su funcionamiento.

Los resultados presentados en la sección 4 demuestran que el IKM obtenido es totalmente comparable, tanto en precisión como en tiempo de cómputo, con los modelos cinemáticos calculados por métodos tradicionales, por lo que el procedimiento desarrollado en este proyecto representa una solución, sistemática e independiente de la geometría del robot, al problema cinemático de sistemas de cadena cinemática abierta.

\section{Agradecimientos}

Los autores desean agradecer la subvención parcial de este trabajo al Plan Nacional de $\mathrm{I}+\mathrm{D}+\mathrm{i}$, Agencia Estatal de Investigación del Ministerio de Economía, Industria y Competitividad del Gobierno de España, por el proyecto FEDER-CICYT DPI2017-84201-R.

\section{English summary}

OBTENTION OF THE INVERSE
KINEMATIC MODEL
OPEN-CHAIN OF
SYSTEMS USING GROEBNER
BASES. APLICATION ON AN
HEXAPOD ROBOT

\begin{abstract}
One of the most important elements of the control system of a robotic system is its Inverse Kinematic Model (IKM), which calculates the position and velocity references required by the robot to follow a predetermined trajectory. The methods that are commonly used to obtain the IKM of open-chain robotic systems strongly depend on the geometry of the analyzed robot and they are not systematic procedures. This work presents the development of a procedure to calculate the IKM of open-chain
\end{abstract}

robotic systems using Groebner Bases. The proposed procedure doesn't depend on the geometry of the studied robot, offering a systematic methodology for the calculation of the IKM. The inputs of the developed procedure are the robot's Denavit-Hartenberg parameters, and it offers as outputs the codes that constitute the calculated IKM. These codes can be used directly in the robot's control system or to simulate its behaviour. The performance of the developed procedure was proved calculating the IKM of a walking hexapod robot. The computation time of the calculated IKM is comparable to the time required by the kinematic model obtained by traditional methods, and the error of the computed references is absolutely negligible in all the hexapod's workspace.

Keywords: Kinematic Problem, Inverse Kinematic Model, Groebner Bases, hexapod walking robot.

\section{Referencias}

[1] Abbasnejad, G., and Carricato, M. "Direct Geometrico-static Problem of Underconstrained Cable-Driven Parallel Robots with n Cables". IEEE Transactions on Robotics 31, 2 (2015), 468-478.

[2] Atique, M. U., Sarker, R. I., And Ahad, A. R. "Development of an 8DOF quadruped robot and implementation of Inverse Kinematics using Denavit-Hartenberg convention". Heliyon 4, 12 (2018).

[3] Barrientos, A., Álvarez, M., Hernández, J. D., del Cerro, J., AND Rossi, C. "Modelado de Cadenas Cinemáticas mediante Matrices de Desplazamiento. Una alternativa al método de Denavit-Hartenberg". RIAI - Revista Iberoamericana de Automática e Informática Industrial 9, 4 (2012), 371-382.

[4] Bouzgou, K., AND Ahmed-Foitin, Z. "Geometric modeling and singularity of 6 DOF Fanuc 200IC robot". In 4th International Conference on Innovative Computing Technology, INTECH 2014 (2014), IEEE, pp. 208-214. 
[5] Chen, S., Luo, M., Abdelaziz, O., AND JiAng, G. "A General Analytical Algorithm for Collaborative Robot (cobot) with 6 Degree of Freedom (DOF)". In Proceedings of the 2017 IEEE International Conference on Applied System Innovation for Modern Technology, ICASI 2017 (2017), IEEE, pp. 698-701.

[6] Cox, D. A., Little, J., And O'SheA, D. "Ideals, Varieties, and Algorithms: An Introduction to Computational Algebraic Geometry and Commutative Algebra", Third ed. Undergradute Texts in Mathematics series. Springer Science, New York, NY 10013, USA, (2007).

[7] DukA, A. V. "Neural Network based Inverse Kinematics Solution for Trajectory Tracking of a Robotic Arm". Procedia Technology 12 (2014), 20-27.

[8] Flanders, M., and Kavanagh, R. C. "Build-A-Robot: Using Virtual Reality to Visualize the Denavit-Hartenberg Parameters". Computer Applications in Engineering Education 23, 6 (2015), 846-853.

[9] Gan, D., Liao, Q., Dai, J. S., Wei, S., And Seneviratne, L. D. "Forward displacement analysis of the general 6-6 Stewart mechanism using Gröbner Bases". Mechanism and Machine Theory 44, 9 (2009), 1640-1647.

[10] Huang, X., And He, G. "Forward Kinematics of the General Stewart-Gough Platform Using Gröbner Basis". In 2009 IEEE International Conference on Mechatronics and Automation, ICMA 2009 (2009), IEEE, pp. 3557-3561.

[11] Jiang, G., Luo, M., Bai, K., And Chen, S. "A Precise Positioning Method for a Puncture Robot Based on a PSO-Optimized BP Neural Network Algorithm". Applied Sciences 7, 10 (2017), 969.

[12] Kendricks, K. D. "A kinematic analysis of the GMF A-510 robot: An introduction and application of Groebner Basis theory". Journal of Interdisciplinary Mathematics 16, 2-03 (2013), 147-169.

[13] KÖKER, R. "A genetic algorithm approach to a neural-network-based inverse kinematics solution of robotic manipulators based on error minimization". Information Sciences 222 (2013), 528-543.
[14] Mahajan, A., Singh, H. P., And SukaVAnam, N. "An unsupervised learning based neural network approach for a robotic manipulator". International Journal of Information Technology 9, 1 (2017), 1-6.

[15] Özgür, E., And Mezouar, Y. "Kinematic modeling and control of a robot arm using Unit Dual Quaternions". Robotics and Autonomous Systems 77 (2016), 66-73.

[16] Petrescu, R. V., Aversa, R., Apicella, A., Mirsayar, M., Kozaitis, S., Abu-Lebdeh, T., And Petrescu, F. I. "Geometry and Inverse Kinematic at the MP3R Mobile Systems". Journal of Mechatronics and Robotics 1, 2 (2017), 58 65.

[17] Rameau, J. F., And Serré, P. "Computing mobility condition using Groebner Basis". Mechanism and Machine Theory 91 (2015), 21-38.

[18] Rodriguez, R., Cardozo, T., Ardila, D. L., And Cuellar, C. A. "A Consistent Methodology for the Development of Inverse and Direct Kinematics of Robust Industrial Robots". ARPN Journal of Engineering and Applied Sciences 13, 1 (2018), 293-301.

[19] Rokbani, N., And Alimi, A. M. "Inverse Kinematics using Particle Swarm Optimization. A Statistical Analysis". In Procedia Engineering (2013), vol. 64, Elsevier, pp. 1602-1611.

[20] Toshani, H., And FArrokhi, M. "Real-time inverse kinematics of redundant manipulators using neural networks and quadratic programming: A Lyapunov-based approach". Robotics and Autonomous Systems 62, 6 (2014), 766-781.

[21] UChidA, T., AND MCPheE, J. "Using Gröbner Bases to generate efficient kinematic solutions for the dynamic simulation of multi-loop mechanisms". Mechanism and Machine Theory 52 (2012), 144-157.

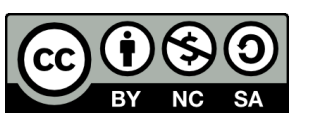
(c) 2019 by the authors. Submitted for possible open access publication under the terms and conditions of the Creative Commons Attribution CC BY-NC-SA 4.0 license (https://creativecommons.org/licenses/by-nc-sa/4.0/ deed.es). 